\title{
Lumen
}

Selected Proceedings from the Canadian Society for Eighteenth-Century Studies

\section{De la ponctuation, à propos de Marivaux}

\section{Raymond Joly}

Volume 20, 2001

URI : https://id.erudit.org/iderudit/1012307ar

DOI : https://doi.org/10.7202/1012307ar

Aller au sommaire du numéro

Éditeur(s)

Canadian Society for Eighteenth-Century Studies / Société canadienne d'étude du dix-huitième siècle

ISSN

1209-3696 (imprimé)

1927-8284 (numérique)

Découvrir la revue

Citer cet article

Joly, R. (2001). De la ponctuation, à propos de Marivaux. Lumen, 20, 123-135.

https://doi.org/10.7202/1012307ar

Copyright $@$ C Canadian Society for Eighteenth-Century Studies / Société canadienne d'étude du dix-huitième siècle, 2001
Ce document est protégé par la loi sur le droit d'auteur. L'utilisation des services d'Érudit (y compris la reproduction) est assujettie à sa politique d'utilisation que vous pouvez consulter en ligne.

https://apropos.erudit.org/fr/usagers/politique-dutilisation/ 


\section{De la ponctuation, à propos de Marivaux}

Ma communication ${ }^{1}$ est née, je l'avoue, d'un agacement: celui que j'éprouve en travaillant avec ces éditions d'auteurs du XVIII ${ }^{\mathrm{e}}$ siècle qui, se conformant à ce qui est en train de devenir l'usage établi, modernisent l'orthographe (attention bien inutile dans une édition savante en tout cas), mais se font gloire de modifier le moins possible la ponctuation de l'auteur. Le motif invoqué est toujours le même: à l'époque, on ponctuait plutôt en fonction de la diction que de la syntaxe ${ }^{2}$, et toucher aux virgules de Marivaux ou de Diderot serait nous priver d'entendre quelque chose du souffle même de l'écrivain.

Or il me semble déceler quelque légèreté dans la façon dont ces raisons sont invoquées et dans la pratique qui en résulte. Je ne suis pas sûr que ce discours ait toujours été précédé d'une réflexion suffisamment méthodique sur ce qu'est la ponctuation, sur la forme qu'elle prenait au XVIII ${ }^{\mathrm{e}}$ siècle et sur le but à poursuivre quand on publie un ouvrage de cette époque ${ }^{3}$.

Rappelons pour commencer l'extrême pauvreté de la ponctuation si on la compare aux inflexions de l'oral. Pour donner une idée un peu juste des variations, en général concomitantes, du timbre de la voix, de la hauteur, de l'intensité, du rythme et du tempo, sans parler de l'éventail des durées des pauses, il faudrait au moins autant de signes qu'il y en a en musique, et la musique en a vingt fois plus qu'il n'existe de signes de ponctuation; de surcroît, elle se note d'une manière qui rend compte de la simultanéité des événements. J'ai entendu jouer la Mer de Debussy dans une réduction pour piano à quatre mains, et c'était magnifique; mais les interprètes auraient été ridicules s'ils avaient prétendu faire deviner l'orchestration en s'adjoignant un violon et deux trombones. Or ce qu'on obtient en pressurant la ponctuation est de cet ordre. Elle n'en est pas moins respectable, mais il convient, précisément, de la respecter plutôt que de lui faire violence en poursuivant un objectif chimérique et qui ne l'est pas moins quand on remonte à des époques dont l'outillage était encore plus rudimentaire que le nôtre. Marivaux, par exemple, manque d'aisance dans l'emploi des parenthèses, ignore complètement 
le tiret et n'imagine pas les nuances que nous faisons rendre aux guillemets et à l'italiqué.

Plus fondamentalement, il ne faut jamais oublier, lorsqu'on aborde ces questions, qu'en présence d'un texte, quel qu'il soit, le lecteur est sourd et l'auditeur, aveugle. Le lecteur ne perçoit aucune modulation de voix, l'auditeur n'entend ni point ni virgule. En un mot - il y a des lapalissades bonnes à rappeler -, la ponctuation n'appartient pas au système de l'oral, mais de l'écrit. Qu'on souligne le rapport étroit qu'elle entretient avec l'éventuelle réalisation orale du texte, c'est énoncer l'évidence; mais il ne faudrait pas s'hypnotiser là-dessus au point de méconnaître la radicale hétérogénéité des deux modes d'utilisation du langage, que la ponctuation, justement, illustre d'une manière frappante.

En effet, les variations d'intonation, de tempo, de volume, de timbre, etc., qui scandent la parole et lui confèrent son expressivité, ne portent presque jamais sur un seul son; elles affectent des segments entiers de la phrase. Et elles ne se produisent pas entre les sons, mais en même temps qu'eux. Au contraire, les signes de ponctuation occupent un espace étroit sur la ligne, avant ou après les lettres, bien qu'eux aussi aient une portée suprasegmentale: ils sont des indices du rapport qu'entretiennent entre eux les segments du discours. L'action de la virgule ou des deux-points ne s'exerce pas au premier chef à l'emplacement que l'écrit leur assigne sur le papier, mais sur ce qui les précède et les suit. Le point final n'est pas une invitation à piquer du nez à la fin de la phrase, le bon lecteur avait préparé sa descente de plus loin. Le point d'interrogation n'ordonne pas non plus à la voix de se retrousser à son aspect, mais d'imprimer à la phrase une courbe interrogative, ce qui très souvent se sera exécuté une ou deux ou même plusieurs lignes plus tôt; cela est si vrai que beaucoup de scripteurs, arrivés à la fin, oublient de mettre le signe interrogatif. Convenons-en: il n'y a sans doute pas de fait de ponctuation, que ce soit la présence ou l'absence d'un signe, qui n'exprime quelque intention susceptible de se manifester à l'oral; mais il est capital de saisir qu'une fraction seulement de cette manifestation se produit, dans la chaîne entendue, à l'endroit où le lecteur aperçoit le signe écrit.

Si les idées anciennes et constamment rabâchées étaient ipso facto justes, il faudrait continuer d'enseigner qu'aux signes de ponctuation doivent correspondre, dans la diction, des pauses. Or cette affirmation est exacte si l'on se réfère aux psautiers et aux lectionnaires des moines qui chantent l'office divin; partout ailleurs, la ponctuation aide à saisir le sens du texte en donnant des indications sur les mots qui vont ou ne vont pas ensemble, sur le rapport entre ces groupes de mots et parfois sur des modalités de l'énonciation. Mais elle ne préjuge pas des moyens à employer pour porter ce sens et ces intentions à l'oreille d'un auditeur. La pause en est un, fréquemment employé mais rarement seul; et la 
signification peut se traduire aussi bien dans l'intonation, le tempo, l'accentuation, etc., que par une coupe. En revanche, la coupe est souvent naturelle, voire indispensable, à des endroits où le papier ne porte que le blanc entre deux mots.

Trois exemples suffiront pour l'illustrer (je ne me doutais pas, en écrivant la première phrase de ma communication, qu'elle resservirait ici):

1. «Mais non!» s'écria-t-elle. [Aucune pause nécessaire.]

2. [...] lorsqu'on travaille avec ces éditions d'auteurs du XVIII ${ }^{\mathrm{e}}$ siècle qui, se conformant à ce qui est en train de devenir l'usage établi, modernisent l'orthographe. [Je lis spontanément en faisant une pause avant "qui» et aucune après; pourtant, la virgule avant la relative déterminative serait absurde, et on ne saurait guère se dispenser $d$ 'encadrer la participiale.]

3. C'est avec une profonde émotion que je vous invite à lever votre verre pour rendre hommage avec moi à un homme en qui tant d'entre nous s'enorgueillissent de saluer aussi bien un maître qu'un ami. [Où voulez-vous mettre une virgule là-dedans? Et imagine-t-on quelqu'un débitant cela d'une traite?]

Concluons que, lorsqu'une phrase paraît mal scandée ou qu'elle ne livre pas son sens du premier coup - parce qu'il y manque une virgule, disons, ou qu'il y en a une de trop -, ce n'est pas trahir l'auteur, son souffle ni sa respiration que d'ajouter la virgule ou de la biffer. Il y aurait sacrilège seulement s'il était établi que la présence ou l'absence du signe témoignent indubitablement d'une intention relative à la réalisation orale (réelle ou mentale) du passage en question; et même dans ce cas, il conviendrait d'accorder la préséance à la limpidité du texte.

En effet, si vous deviez induire de ce que vous venez d'entendre que je prêche la ponctuation dite «syntaxique» au nom de la logique de l'écrit et aux dépens de la réalisation orale des textes, vous vous tromperiez du tout au tout. C'est par amour de l'oral que je suis partisan de la logique de l'écrit. Il m'est si impossible de m'imaginer la littérature comme faite pour l'œil que je dirais volontiers indifféremment «le lecteur» ou «l'acteur» pour désigner celui au profit de qui on réédite Marivaux — ou n'importe quel autre écrivain, à l'exception de ceux de nos contemporains qui attribuent une fonction structurante à la disposition du poème sur le papier. Bien lire une pièce de théâtre exige qu'on se représente les répliques comme adressées à un autre personnage, donc comme dites. Lire un roman de Diderot ou un discours de Rousseau, c'est écouter une voix qui cherche à nous atteindre, dans le coin d'un salon ou sur un forum imaginaire; lire Madame Bovary ou À la recherche du temps perdu, c'est suivre Flaubert et Proust dans leur gueuloir ou dans la chambre de liège où ils ont convoqué un ami pour lui parler jusqu'à cinq heures du 
matin. C'est pour que la phrase vienne bien en bouche que la ponctuation doit aider à en saisir immédiatement la structure. Ensuite seulement on peut s'occuper des effets expressifs. Avant de songer à des rallentando, à des mezza voce, à des con espressione et à des leggiero, la cantatrice s'assure de connaître exactement la durée de chaque note et sa place dans la mesure.

On a voulu distinguer une ponctuation "pour l'œil» et une ponctuation "pour l'oreille»: l'oreille décline un tel excès d'honneur, car elle restera toujours insensible à la lumière. Si l'on veut absolument une opposition frappante, tournons-nous plutôt du côté où les choses se passent vraiment, observons le lecteur dont la vue est affectée par les signes écrits et opposons une ponctuation «pour l'œil» à une ponctuation "pour la bouche et la poitrine». Cela nous fera immédiatement apercevoir qu'il n'y en a en vérité qu'une seule: la ponctuation pour l'esprit par le canal de l'œil. C'est le travail du premier, interprète des indices relevés par le second, qui rend possible la métamorphose, mentale ou concrète, de l'écrit en oral. Selon le tempérament et le talent de chacun (ou sa profession), selon le genre de texte qu'il lit et l'intention dans laquelle il lit, intérieurement il animera plus ou moins l'écrit de la chaleur de sa voix; il pourra même finir par le projeter réellement hors de son corps sous forme d'ondes acoustiques. Mais malheur à lui s'il fait des effets de voix avant d'avoir compris ce qu'il disait. Et il aura le droit de se fâcher si la ponctuation l'a mis sur de fausses pistes.

La mode de la «ponctuation orale» n'afflige pas seulement les auteurs anciens, et je ne saurais mieux résumer mon propos qu'en commentant quelques lignes, très typiques de ce courant, relevées chez un récent éditeur de Proust. Si l'auteur de la Prisonnière «n'omet pas de placer des points entre les phrases», lisons-nous sous cette plume, «il utilise beaucoup moins les autres signes et, lorsqu'il le fait, il se conforme avant tout à l'usage du débit oral, qui se sert plus fréquemment des variations de l'intonation que des pauses comme procédés démarcatifs $[\ldots]^{5}$ » (éd. Milly, p. 82). Il se trouve malheureusement que le débit oral ne connaît ni le point final ni les autres signes et qu'il ne saurait donc suivre quelque usage que ce soit en la matière, ce qui rend tout à fait absurde l'idée qu'un écrivain le prenne pour modèle. D'autre part, notre éditeur a très bien vu que les inflexions vocales autres que la pause jouaient un rôle décisif dans la structuration du discours parlé, mais la fidélité au préjugé scolaire selon lequel ponctuation est synonyme de pauses ou de coupures (il emploie les deux termes) l'empêche de tirer profit de son observation. À la fin, il débouche sur un cercle vicieux pur et simple: «Il y a sans doute eu un excès pédagogique à fragmenter ses phrases [celles de Proust] en ajoutant un grand nombre de virgules et de points-virgules, à l'usage d'une lecture scolaire». Or, quand les virgules et les points-virgules 
suscitent une lecture scolaire et fragmentée plutôt qu'une lecture vivante, souple et nuancée, c'est parce que le lecteur obéit au maître d'école qui a décrété que les virgules et les points-virgules signifiaient stop. L'objectif à viser est tout autre, et j'inscris avec enthousiasme mon accord: il faut permettre de lire Proust, et la Vie de Marianne, comme «une sorte d'immense monologue où doivent être nécessairement rétablies par l'imagination du lecteur les inflexions de la voix». Le meilleur moyen de l'en empêcher est de l'astreindre à s'échiner sur une syntaxe devenue énigmatique par la faute d'une ponctuation insuffisante, équivoque ou à contresens.

Les idées que je viens de développer relèvent, me semble-t-il, du sens commun et naissent spontanément pour peu qu'on ait prêté attention quelquefois à la façon dont les bons lecteurs (comédiens ou autres) s'y prennent pour donner vie à l'écrit. Par ailleurs, elles ne sont nullement contredites par les études de linguistique et de psycholinguistique publiées depuis une trentaine d'années sur la ponctuation, sur l'oral et sur les rapports entre les deux. On en trouvera quelques-unes ci-dessous dans la bibliographie; elles ne sont pas toutes sans enseignements pour notre confrérie de littéraires. Je vois avec plaisir, dans un bel article sur les applications pédagogiques de ces recherches, que l'une des tâches des enseignants est «de faire remarquer au passage à leurs élèves que leur segmentation pausale à l'oral ne reproduit pas le marquage de la ponctuation à l'écrit» (Bessonnat, p. 13). Partirons-nous en croisade contre une école qui vole à ses pupilles leur «souffle» et leur «respiration»? Bien au contraire, elle les leur rend en libérant la parole et l'écrit de l'esclavage réciproque où les réduisait une conception erronée de leur correspondance en miroir.

Si j'évoquais tout à l'heure la délicate décision qu'a à prendre l'éditeur placé devant une ponctuation aberrante dans un texte d'un écrivain de notre siècle, qu'en sera-t-il de celui qui travaille sur un contemporain de Louis XV ! En effet, dans le premier cas, l'éditeur est en droit de supposer que son auteur attribue en principe la même valeur que lui aux signes de ponctuation. Rien n'autorise pareille confiance si on remonte dans le passé. Il est étonnant de voir de bons érudits d'aujourd'hui se comporter comme si une virgule était une virgule et les deux-points, des deuxpoints. Cela est aussi aberrant que de soutenir que le $s$ final des substantifs est en français la marque du pluriel alors que, jusque fort avant dans le XIII ${ }^{\mathrm{e}}$ siècle, une bonne moitié des noms prenaient justement l's au singulier pour le perdre au pluriel quand ils étaient en fonction de sujet. Et arguera-t-on que dessein, dans un livre du XVIII ${ }^{\mathrm{e}}$ siècle, signifie néces- 
sairement «dessein» puisqu'il n'est pas écrit dessin? La ponctuation est un sous-ensemble du code écrit et elle fonctionne comme tout ce qui est langue, c'est-à-dire que la valeur de ses éléments à une époque donnée dépend tout entière du jeu de leurs oppositions dans le cadre du système tel qu'il s'organise à l'époque en question.

Le code de la ponctuation au XVIII ${ }^{\mathrm{e}}$ siècle, qui est encore plutôt mal connu, diffère du nôtre dans des proportions considérables. Mon premier exemple fait comprendre le désespoir de l'acteur ou de l'éditeur devant ce qui relève peut-être bien de l'indécidable; les suivants mettent en lumière $\mathrm{d}$ 'une autre façon les pièges de la fidélité.

4. La Demoiselle de Paris qui s'était travestie en chevalier vient d'être démasquée d'une manière qui ne laisse plus de place à l'esquive. Elle doit avoir le caquet bien bas? Au contraire:

La Fausse Suivante, p. 115 Théâtre, éd. DeloffreRubellin, I, p. 459

Moi déconcertée! pas un petit brin; graces au Ciel! je suis une femme, et je soûtiendrai mon caractere.
Moi déconcertée! pas un petit brin, grâces au ciel; je suis une femme, et je soutiendrai mon caractère.
Théâtre, éd. Coulet-Gilot, I, p. 364

Moi déconcertée! pas un petit brin, grâces au ciel! je suis une femme, et je soutiendrai mon caractère.

Si l'auteur vivait aujourd'hui, il aurait sans doute opté pour l'une des rédactions suivantes:

5. Moi déconcertée! Pas un petit brin, grâce au ciel! Je suis une femme, et je soutiendrai mon caractère. [C'est ce qu'ont compris Deloffre et Rubellin, et apparemment Coulet et Gilot; autrement on ne comprendrait pas ce qui leur a fait remplacer eux aussi le point-virgule par une virgule malgré leur fidélité affichée à la ponctuation originale, position qui en revanche leur impose de maintenir le second point d'exclamation ${ }^{6}$.]

6. Moi déconcertée! Pas un petit brin. Grâce au ciel, [ou Grâce au ciel!] je suis une femme, et je soutiendrai mon caractère.

Selon moi, le texte de l'originale, dans un livre paru aujourd'hui, s'interpréterait spontanément dans le sens de la version 6: la minuscule au premier mot de «je suis une femme», en dépit du point d'exclamation qui précède, prouve que la phrase ne commence pas là et que la proposition se relie à "grâce au ciel», tandis que le point-virgule marque une frontière nette entre ces trois mots et "pas un petit brin». Mais ce raisonnement ne nous mène pas avec certitude vers l'interprétation 
authentique de ce qu'écrivit Marivaux, car il repose sur une conception précise de la valeur de deux signes, la majuscule et le point-virgule, dont la signification était beaucoup plus flottante à son époque.

De nos jours, la fonction typique du point-virgule consiste à séparer des énoncés complets, indépendants au point de vue de la syntaxe, et néanmoins assez étroitement liés quant à l'intention du discours pour $q^{\prime}$ 'on veuille le faire sentir ${ }^{7}$. Sous Louis XV, ce n'était souvent pas autre chose qu'une virgule forte, et on le trouve couramment entre la principale et la subordonnée, voire entre la subordonnée et la principale. Par ailleurs, le XVIII ${ }^{\mathrm{e}}$ siècle n'avait pas encore codifié une marque ponctuationnelle devenue pour nous élémentaire et fondamentale: la majuscule en tête de phrase. Dans les éditions originales de Marivaux, la majuscule après le point-virgule, les deux-points ou la virgule ne prouve pas qu'une phrase commence, et certains débuts de phrase indubitables en sont dépourvus. De là naissent mille ambiguïtés, et on ne saurait nier qu'elles soient imputables dans une large mesure à l'incurie; bonne chance à qui entreprendra par exemple de justifier les deux occurrences, en contexte totalement dissemblable, de point-virgule - alinéa - majuscule à la page xxxvi de l'Homère travesti. Mais nous aurions tort de nous scandaliser trop hautement. D'abord, notre conception de la phrase nos conceptions de la phrase - ne sont pas un monument de logique et de netteté du haut duquel nous puissions regarder avec condescendance les tâtonnements des siècles passés. Ensuite, avant d'exiger des majuscules au début des phrases, il faudrait nous assurer que nos interlocuteurs savent ce que nous voulons dire. Or, à l'époque qui nous intéresse, ceux qui parlaient de ponctuation dissertaient sur le discours, sur la période et la proposition, sur les sens plus ou moins partiels, suspendus, complets ou terminés qu'il fallait distinguer, et non sur la phrase. En effet, les faits de langue qu'évoquait pour eux ce terme, bien plus proche de l'anglais phrase que des tentatives de définition des syntacticiens modernes, relèvent de ce que nous appelons stylistique et, précisément, phraséologie.

Voici maintenant quelques exemples d'un point-virgule que Marivaux affectionnait. Cet emploi heurte de plein fouet la doctrine unanime de ses contemporains, il est fort gênant aujourd'hui et l'était probablement de son temps déjà.

7. L'oncle et la nourrice de Pharsamon vont réveiller le valet qu'ils avaient ligoté la veille, le croyant fou:

Ce qui fut conseillé fut fait, ils vont tous deux trouver Colin qui malgré ses menottes, n'avoit pas laissé que de céder aux doux charmes de Morphée, on l'avoit long-temps gardé à vûë; mais le profond sommeil qui l'avoit gagné, avoit 
fait songer à celui qu'on avoit mis à sa garde, qu'il seroit inutile de le veiller davantage (Pharsamon, p. 104).

Le sens demande d'intervertir le point-virgule et la virgule qui le précède, comme dans les deux extraits qui vont suivre.

8. Les romans ont appris à Cliton avec quel art les princesses provoquaient des rencontres providentielles, et avec quelle hauteur elles repoussaient les amants irrespectueux. La réalité l'a profondément déçu:

toutes les Demoiselles d'alentour nous paroissoient au prix, de vraies cuisinieres, il n'y en a pas une qui ait eu l'esprit d'aller dormir dans les bois ou dans un pavillon, au lieu de crier quand on leur baise la main; elles vous rient au nez comme des sottes (Pharsamon, p. 117).

9. Lelio s'adresse à la Comtesse. C'est le second point-virgule qui est instructif: Je ne pense point que vous m'aimez, Madame, vous me traitez mal, mais vous y trouvez du goût: N'usez point de prétexte, je vous ai déplû d'abord; moi specialement je l'ai remarqué, et si je vous aimois; de tous les hommes qui pourroient vous aimer, je serois peut-être le plus humilié, le plus raillé, et le plus à plaindre (la Surprise de l'amour, p. 74).

Ces trois exemples donnent une idée du fonctionnement de ce que j'appellerai le point-virgule agrafe. La règle s'en formule ainsi: Dans une phrase qui comporte deux ou plusieurs éléments $A, B, C$ de même niveau, coordonnés ou juxtaposés, lorsque $C$ se décompose lui-même en $c^{1}$ et $c^{2}$ mutuellement dépendants au point de vue de la syntaxe ou du sens, Marivaux marque volontiers ce lien au moyen du point-virgule ${ }^{8}$.

Le lecteur moderne reste estomaqué et, comme je l'ai dit, nos ancêtres ressentaient probablement eux aussi de l'agacement, car il y avait déjà quelques siècles, en 1730 , que le point-virgule se situait à un niveau hiérarchique supérieur à la virgule, séparait des éléments moins étroitement liés et marquait donc une césure plus importante, ce qui entraînait des conséquences dans la manifestation acoustique du sens, réelle ou imaginée. Les phrases qu'on vient de lire sont bancales, obscures ou absurdes tant qu'on ne les a pas reponctuées, réellement ou mentalement ${ }^{9}$. Le temps ni le lieu ne se prêtent à la multiplication des exemples, et je dois vous demander de me faire confiance: cela se retrouve à tout bout de champ chez Marivaux et appartient à sa ponctuation régulière.

Mais ce n'est pas tout. On s'étonne déjà en butant sur des pointsvirgules qui s'avèrent plus faibles que les virgules précédentes. Or il arrive par-dessus le marché que le point-virgule agrafe pâlisse encore devant la virgule qui le suit. En effet, cette pauvrette, l'auteur lui confie parfois une tâche bien lourde et bien peu conforme à sa nature: celle 
$\mathrm{d}^{\prime}$ indiquer une réorientation du discours ${ }^{10}$. Voilà ce qu'on observe dans mon dernier exemple:

10. La Castille est en pourparlers avec Barcelone. L'ambassadeur de Castille laisse entendre que son maître sait rémunérer les entremetteurs complaisants, ce qui lui attire de la part de son vis-à-vis cette énergique rebuffade:

\section{Le Prince travesti, p. 85}

Ah de grace, ne citez point ici le Roy votre Maître, soupçonnezmoi tant que vous voudrez de manquer de droiture; mais ne l'associez point à vos soupçons, quand nous faisons parler les Princes, Monsieur, que ce soit toujours d'une maniere noble et digne d'eux $[\ldots]$.
Théâtre, éd. DeloffreRubellin, I, p. 373

Ah! de grâce, ne citez point ici le Roi votre maître; soupçonnez-moi tant que vous voudrez de manquer de droiture, mais ne l'associez point à vos soupçons. Quand nous faisons parler les princes, Monsieur, que ce soit toujours d'une manière noble et digne d'eux [...].
Théâtre, éd. Coulet-Gilot, I, p. 293

Ah de grâce, ne citez point ici le roi votre maître, soupçonnez-moi tant que vous voudrez de manquer de droiture; mais ne l'associez point à vos soupçons. Quand nous faisons parler les princes, Monsieur, que ce soit toujours d'une manière noble et digne d'eux $[\ldots]$.

Même les éditeurs de la Pléiade n'y ont pas tenu, et ils ont mis le point indispensable. Mais que penser du reste? À quelle logique obéissent les autres signes qu'ils maintiennent? Certes pas à celle d'aujourd'hui. Et est-il raisonnable de présumer que l'amateur de littérature et le comédien, aux alentours de l'an 2000, reconnaissant au premier coup d'œil le point-virgule agrafe, intervertiront spontanément les signes pour retrouver l'intonation juste?

Je rappellerai, pour conclure, les trois partis qui s'offrent, au point de vue qui nous intéresse ce matin, lorsqu' on publie un ouvrage du XVIII ${ }^{\mathrm{e}}$ siècle.

Le premier est le plus simple à prendre, c'est l'édition diplomatique: on reproduit tout simplement l'original tel quel, en ne corrigeant, avec prudence, que les coquilles évidentes. Je suggérerais alors une précaution. Il serait sage, si on ne vise pas uniquement un public de spécialistes, de lui expliquer un peu comment fonctionne la ponctuation du temps, afin de dissiper certains étonnements et surtout d'éviter des contresens. 
Pour ne mentionner que des particularités sur lesquelles j'ai vu les étudiants trébucher, et même parfois d'éminents collègues, il faudrait signaler que le point d'interrogation signifie à l'occasion bien autre chose que ce que son nom indique; prévenir que le point-virgule à la fin d'une proposition peut équivaloir à la parenthèse fermante, ce qui impose d'en imaginer rétroactivement quelque part une ouvrante, ou l'inverse; mettre en garde contre la tentation de croire que l'énoncé qui suit les deux-points est l'explication de ce qui précède; préparer ses lecteurs à devoir rétablir le rapport logique entre principale et subordonnée en ramenant bien des points-virgules à de plus modestes proportions, etc. Je voudrais aussi qu'on soit cohérent. Je n'en reviens pas lorsque tel savant éditeur, qui dispose de manuscrits et d'imprimés, se scandalise à l'idée de reproduire l'orthographe de son auteur et celle de ses libraires, sous prétexte qu'elles ne sont pas en tout point identiques et que leur juxtaposition produirait une sorte d'«habit d'Arlequin», et qu'on voit ensuite le même homme, oublieux des conseils de l'Évangile, coudre ensemble la ponctuation d'il y a deux cents ans et le tissu tout neuf de son orthographe modernisée. Les losanges multicolores d'Arlequin, au moins, ne sont pas anachroniques.

Le second parti possible consiste à moderniser la ponctuation aussi, et c'est sans doute le meilleur si l'on vise un autre public que l'ordre des dix-huitiémistes de la stricte observance. Tâche redoutable, comme l'est toute traduction. Car c'est traduire que de reformuler selon un certain code un message qui avait été rédigé selon un autre ${ }^{11}$. Et la difficulté n'est pas moindre lorsque, comme ici, les signifiants du code de départ sont les mêmes que ceux du code d'arrivée. Quand trahit-on le plus? Lorsqu'on remplace tels deux-points par un point-virgule, ou lorsqu'on les laisse tout en sachant qu'ils n'ont plus la valeur que Marivaux leur attribuait? Les traducteurs appellent faux amis les mots qui se retrouvent dans la langue de départ et dans la langue d'arrivée, mais avec un sens différent dans chacune: la ponctuation d'un livre du XVIII siècle est un chapelet de faux amis.

La troisième façon de faire combine et aggrave les inconvénients des deux précédentes, ce qui explique sans doute sa popularité. Par fidélité au fameux souffle de l'écrivain, par admiration pour la mythique oralité de la ponctuation d'autrefois, on la reproduit, mais en la retouchant quand même un peu, c'est-à-dire pas mal souvent, quand elle risquerait de déconcerter par trop le lecteur moderne. Et que fera-t-il donc, ce malheureux? Tentera-t-il de dégager par lui-même la logique de la ponctuation ancienne? Il n'y arriverait pas, puisqu'on lui en présente un spécimen trafiqué, expurgé des particularités les plus frappantes et peut-être les plus instructives. Lira-t-il suivant le système auquel il est habitué et qu'il pratique tous les jours? Il risque alors de marcher de 
perplexités en contresens puisque le gros de ce qu'on lui met sous les yeux obéit aux règles d'un autre siècle. S'il interprète au contraire à partir d'une connaissance préalablement acquise de la ponctuation ancienne, il a des chances de s'égarer tout autant, puisqu'il lit un texte contaminé par l'usage moderne. Nous lui conseillerons donc de se débrouiller comme il peut en faisant comme si l'édition qu'il a entre les mains n'était pas ponctuée du tout.

\section{RAYMOND JOLY}

\section{Bibliographie}

Bessonnat, Daniel, «Enseigner la... 'ponctuation'? (!)», dans Pratiques, 3-45.

Catach, Nina, la Ponctuation, Paris, Presses universitaires de France, coll. "Que sais-je?», 2818, 1994.

Catach, Nina et Claude Tournier (édit.), la Ponctuation. Recherches historiques et actuelles, Paris et Besançon, Centre national de la recherche scientifique - Groupement de recherches sur les textes modernes - H.E.S.O., 1977-1978, 2 vol.

Defays, Jean-Marc, Laurence Rosier et Françoise Tilkin (édit.), À qui appartient la ponctuation? Actes du colloque de Liège (1997), Paris et Bruxelles, Duculot, coll. «Champs linguistiques», 1998.

Drillon, Jacques, Traité de la ponctuation française, Paris, Gallimard, coll. «Tel», 177, 1991.

Langue française, 45, février 1980: la Ponctuation.

Langue française, 81, février 1989: Structurations de textes. Connecteurs et démarcations graphiques.

Marivaux $^{12}$, la Fausse Suivante, ou le Fourbe puny, Paris, Prault, 1729 (Yf 7259).

Marivaux, l'Homere travesti, ou l'Iliade en vers burlesques, Paris, Prault, t. I, 1716 (Yb 1156).

Marivaux, Pharsamon, ou les Nouvelles Folies romanesques, Paris, Prault, $1737\left(16^{\circ} \mathrm{Y}^{2}\right.$ 17871).

Marivaux, le Prince travesti, ou l'Illustre Avanturier, Paris, Pissot, 1727 ( $8^{\circ}$ Yth 14708).

Marivaux, la Surprise de l'amour, Paris, Gandouin, 1723 (Yf 5843).

Marivaux, Théâtre complet, éd. Henri Coulet et Michel Gilot, Paris, Gallimard, coll. «Bibliothèque de la Pléiade», vol. I, 1993.

Marivaux, Théâtre complet, éd. Frédéric Deloffre et Françoise Rubellin, Paris, Dunod, coll. «Classiques Garnier», vol. I, 1996 (1968).

Marivaux, la Voiture embourbée, Paris, Prault, 1714 (microfilm, localisation de l'original inconnue).

Pratiques, 70, juin 1991: la Ponctuation.

Proust, Marcel, la Prisonnière, éd. Jean Milly, Paris, Flammarion, coll. «GF», 376, 1984. 


\section{Notes}

1 On comprendra à la lecture pourquoi je lui ai conservé sa forme orale.

2 Ce point, déjà, mériterait vérification. Que les théoriciens du temps réfèrent sans cesse la ponctuation aux «pauses» ne garantit pas l'exactitude et l'exhaustivité de leur analyse de l'oral ni de l'écrit. Et il faudrait mettre en balance les cas qui appuient l'idée reçue avec ceux qui dénotent au contraire la surdité du code ponctuationnel - ou son indifférence - à l'endroit des faits de parole. Pensons par exemple au traitement fort peu différencié des relatives explicatives et déterminatives, et à la virgule devant $e t$.

3 Je laisse de côté la question de savoir si ce que nous transmettent les imprimés est le «souffle» de l'écrivain, d'un copiste ou du prote. On concédera facilement que, toutes choses égales d'ailleurs, la ponctuation de l'édition originale est probablement celle qui se rapproche le plus de celle de l'auteur. Précisons également que les écrits intimes, les lettres par exemple, mériteraient un traitement à part. À l'époque, on s'y permettait encore plus de désinvolture qu'aujourd'hui. Quant à accorder à ces négligences les honneurs de l'impression... Les scripteurs en cause se seraient jugés trahis tous les premiers s'ils s'étaient vus présentés au public avant d'avoir pu se donner un coup de peigne.

4 Sauf erreur, les éditions originales de ses pièces publiées dans les années 1720 offrent un seul exemple de l'italique appliqué à une citation de parole (la Fausse Suivante, p. 61), et aucun des guillemets. Et Dieu sait quel usage infiniment varié il faisait de ces reprises de mots ou d'expressions! Il ne lui aurait sans doute pas déplu d'en signaler à l'occasion le statut particulier, s'il avait su comment, puisqu'il s'est donné une fois la peine d'inscrire cette lourde didascalie: «Lelio, repetant un mot» (ibid., p. 46; acte II, sc. 2).

5 Ponctuation impeccable, soit dit en passant. Dommage qu'aucun enregistrement ne nous fasse entendre la voix de l'auteur; il serait intéressant d'observer le nombre et la durée des pauses avant et après «et».

6 Ces remarques valent, bien sûr, dans la supposition que leur exemplaire de l'édition de 1729 appartenait au même état que le mien. - Je n'ai pas voulu faire écrire "Grâces au ciel» à mon contemporain imaginaire. Il va de soi que les éditeurs, eux, ne pouvaient pas ne pas conserver cette graphie, qui témoigne à tout le moins d'un fait de phraséologie, et peut-être de phonétique aussi, même en prose.

7 Cela décrit assez bien les deux-points du XVIII siècle.

8 Avec les plus expresses mises en garde, j'émettrai l'hypothèse que nous avons affaire ici à un cas particulier d'une règle plus universelle, le point-virgule tendant à se définir chez Marivaux comme une marque d'intonation montante, indépendamment de toute considération syntaxique et avec des effets de sens divers.

9 Coulet et Gilot eux-mêmes ont reculé devant le point-virgule agrafe de l'exemple 9 («si je vous aimois ;»); ils mettent une virgule (Théâtre, I, p. 172).

10 L'exemple le plus stupéfiant est sans doute cet endroit de la Voiture embourbée (p. 83) où la virgule qui suit le point-virgule agrafe équivaut à «Point, à la ligne» et marque (je parle par antiphrase) la frontière entre le corps du récit et une apostrophe du narrateur à son destinataire. Les éditions modernes ne laissent entrevoir rien de cela, ce que je ne leur reproche nullement. 
11 Moderniser l'orthographe aussi est traduire. La plupart des transformations se font automatiquement, mais d'autres exigent qu' on opte entre diverses possibilités, donc qu'on interprète.

12 Cotes des éditions originales à la Bibliothèque nationale, Paris. 